\title{
The Effect of Pre-Exercise Cooling on Performance Characteristics: A Systematic Review and Meta-Analysis
}

\author{
Erich Hohenauer 1,2,3, Rahel Stoop ${ }^{1}$, Peter Clarys ${ }^{3}$, Ron Clijsen ${ }^{1,2,3}$, Tom Deliens ${ }^{3}$, Jan Taeymans ${ }^{3,4}$ \\ ${ }^{1}$ Research Rehabilitation 2rLab, Department of Business Economics, Health and Social Care, University of Applied Sciences and \\ Arts of Southern Switzerland, Landquart, Switzerland \\ ${ }^{2}$ THIM University of Applied Sciences, Landquart, Switzerland \\ ${ }^{3}$ Department of Movement and Sport Sciences, Vrije Universiteit Brussel, Brussels, Belgium \\ ${ }^{4}$ Health Department, Bern University of Applied Sciences, Berne, Switzerland \\ Email: erich.hohenauer@supsi.ch
}

How to cite this paper: Hohenauer, E., Stoop, R., Clarys, P., Clijsen, R., Deliens, T. and Taeymans, J. (2018) The Effect of Pre-Exercise Cooling on Performance Characteristics: A Systematic Review and Meta-Analysis. International Journal of Clinical Medicine, 9, 117-141.

https://doi.org/10.4236/ijcm.2018.93012

Received: February 12, 2018

Accepted: March 4, 2018

Published: March 7, 2018

Copyright $\odot 2018$ by authors and Scientific Research Publishing Inc. This work is licensed under the Creative Commons Attribution International License (CC BY 4.0).

http://creativecommons.org/licenses/by/4.0/

\section{Open Access}

\begin{abstract}
Exercising in high environmental temperatures may cause precocious hyperthermia induced fatigue resulting in a decreased athletes' performance output. This systematic review with meta-analysis investigated the possible effects of pre-exercise cooling on performance output. This study was performed according to the PRISMA guidelines and the PICO-model was used to establish the research question. The Cochrane Risk of Bias Tool was applied to assess the validity of the included studies. Study eligibility was given when the studies compared the effects between any kind of pre-cooling and non-cooling strategies prior to exercise on performance output. Twenty-nine studies met the inclusion criteria for quantitative analysis. Risk of bias was high or unclear but the performance bias was low. The estimated standardized mean difference revealed that external pre-cooling (21 studies) enhanced performance (Hedges' $\mathrm{g}=0.49$ [95\% CI: 0.33 to 0.64$]$ ), with the main effect observed in endurance cycling or running. Internal (7 studies) and mixed-method (5 studies) pre-cooling failed to significantly affect performance parameters. However, the main output parameter, evaluated in these studies, was peak power output. Subgroup analysis for different outcome measures was not possible because meaningful grouping was not plausible. Limitations of this meta-analysis were the high or unclear risk of bias and the comparability of the included studies. Future studies should also determine the effects of different pre-cooling applications on female and untrained participants. Based on the results of this meta-analysis, it can be concluded that there is some evidence in favour of external pre-cooling to avoid precocious hyperthermia
\end{abstract}


induced fatigue in endurance athletes exercising in hot environments.

\section{Keywords}

Performance Cooling, Precooling, Core Temperature, Exercise, Meta-Analysis

\section{Introduction}

High environmental temperatures may negatively influence the athletes' performance output due to precocious hyperthermia induced fatigue [1] [2]. Various studies have demonstrated that a hot environment is an important factor which may significantly reduce athletes' power output, especially in endurance sport types such as running sports [3] and cycling [4] [5]. At environmental conditions above $30^{\circ} \mathrm{C}$, core temperature over $40^{\circ} \mathrm{C}$ and coinciding increased brain temperature, performance will be impaired [6] [7] [8] [9]. Under these circumstances, the athlete's thermoregulatory system will try to decrease core temperature to maintain physical performance. The most effective way of heat dissipation from the body is the evaporation of sweat, also in case of systemic diseases [10]. Therefore, the thermoregulation depends strongly on sweat production and sweat evaporation from the skin [11] [12]. Nybo et al. (2014) comprehensively reviewed the existing literature of the last century on the physiological mechanisms that lead to hyperthermia induced fatigue. The authors concluded that various interdepending factors, such as psychological factors, respiration, peripheral and central fatigue factors, neurobiological changes and cardiovascular changes may lead to precocious fatigue during prolonged exercise in the heat [1]. To combat the debilitating effects of hyperthermia induced fatigue, athletes were encouraged to acclimatize to hot environmental conditions [13]. Another strategy to prevent hyperthermia induced fatigue is pre-cooling the body before exercising in the heat. The latter has been demonstrated to increase the heat storage capacity before a critical, performance limiting temperature is reached [14] [15]. As the temperature gradient between the body core and the skin narrows during exercise, blood flow of the skin must be increased to maintain the heat transfer [16] [17]. Additionally, heart rate must be increased for an adequate cardiac output [18]. Consequently, an artificially increased margin between the core temperature and the environmental temperature might be an important factor that leads to an advantage from pre-cooling on performance [19]. Another performance limiting factor seems to be the impaired cardiovascular function to maintain adequate oxygen delivery to the working muscles [18] [20]. Beside reduced metabolism, the increase of intramuscular temperature might lead to impaired mitochondrial functioning, disturbances of $\mathrm{K}^{+}, \mathrm{Ca}^{2+}$ and phosphate homeostasis which leads to decreased muscle contractility [21] [22] [23]. Pre-cooling to maintain or enhance sports performance, is a practical on-field strategy for athletes because of its easy implementation during the 
warm-up period. Various forms of pre-cooling are already practiced and scientifically investigated. External pre-cooling strategies aim to reduce core temperature by decreasing skin and muscle temperature, including the use of ice-vests [24], cold-packs [25], cold-water immersion (CWI) [26] and combinations of cooling different body parts [27]. Internal pre-cooling aims to reduce core temperature and can be performed by ingesting cold water [28] or crushed ice [29] while mixed-method pre-cooling usually contains the combination of ingesting cold water or ice plus an additional partial body cooling application [30]. In a systematic review, Ross et al. (2013) evaluated the effectiveness of pre-cooling during field-based sports performance [31]. The findings of this study are in line with another meta-analytical review from Wegmann et al. (2012) who indicated that the effects of pre-cooling on performance output are larger in hot $\left(>26^{\circ} \mathrm{C}\right)$ environmental conditions than under moderate $\left(18^{\circ} \mathrm{C}\right.$ $26^{\circ} \mathrm{C}$ ) temperatures [32]. Although the bulk of literature is growing in this field, a consensus about which cooling method is the most effective [28] and which performance parameter is the most affected under hot temperature conditions is still lacking [2]. Therefore, the aim of this study was to systematically search and critically evaluate the current literature on the effects of external cooling, internal cooling and mixed-method cooling prior to exercise in comparison to non-cooling strategies on different performance parameters of humans exercising under "hot" as well as under "hot and humid" environmental conditions.

\section{Methods}

\subsection{Research Question}

The research question was defined by the PICO-model in accordance with the Preferred Reporting Items for Systematic Reviews and Meta-Analyses (PRISMA) statement [33]: Population: healthy, non-injured athletes and active participants; Intervention: external cooling or internal cooling or mixed-method cooling (combination of external and internal cooling) prior to a specified exercise protocol; Comparator: passive non-cooling strategies; Outcomes: time to finish, time to exhaustion, speed, covered distances and peak power output (PPO).

\subsection{Literature Search Strategies and Data Sources}

An electronic systematic search, according to the guidelines of the Preferred Reporting Items for Systematic Reviews and Meta-Analyses (PRISMA) statement, was conducted between October 2016 and January 2017 on the MEDLINE (PubMed) database [34]. Studies were included when meeting the following criteria: 1) internal cooling or external cooling or mixed-method cooling was conducted prior to a specified exercise protocol, 2) the cooling strategies had to be compared with a passive strategy that did not use any kind of cooling procedure, 3) hot environmental conditions $\left(>26^{\circ} \mathrm{C}\right)$, 4) participants were healthy humans without any physical infirmity, 5) male and female study participants were included, 6) written in English or German, 7) written full-text studies had to be 
published within the last 10 years $(2006-2016), 8)$ the studies had to be randomized controlled trials (RCT) or randomized cross-over trials (RCO). The keywords which best fitted the research question were: "Pre-cooling", "Precooling", "Cold", "Performance" and "Exercise". These keywords were inserted as follows: ("Pre-cooling" OR "Precooling" OR "Cold") AND "Performance" AND "Exercise". The keywords "Pre-cooling", "Precooling" and "Cold" were used individually, in any order and always combined with ("Performance" AND "Exercise"). After screening and processing all the articles found $(\mathrm{k}=668)$, a total of 29 articles were eligible as sources of primary literature for this meta-analysis. Figure 1
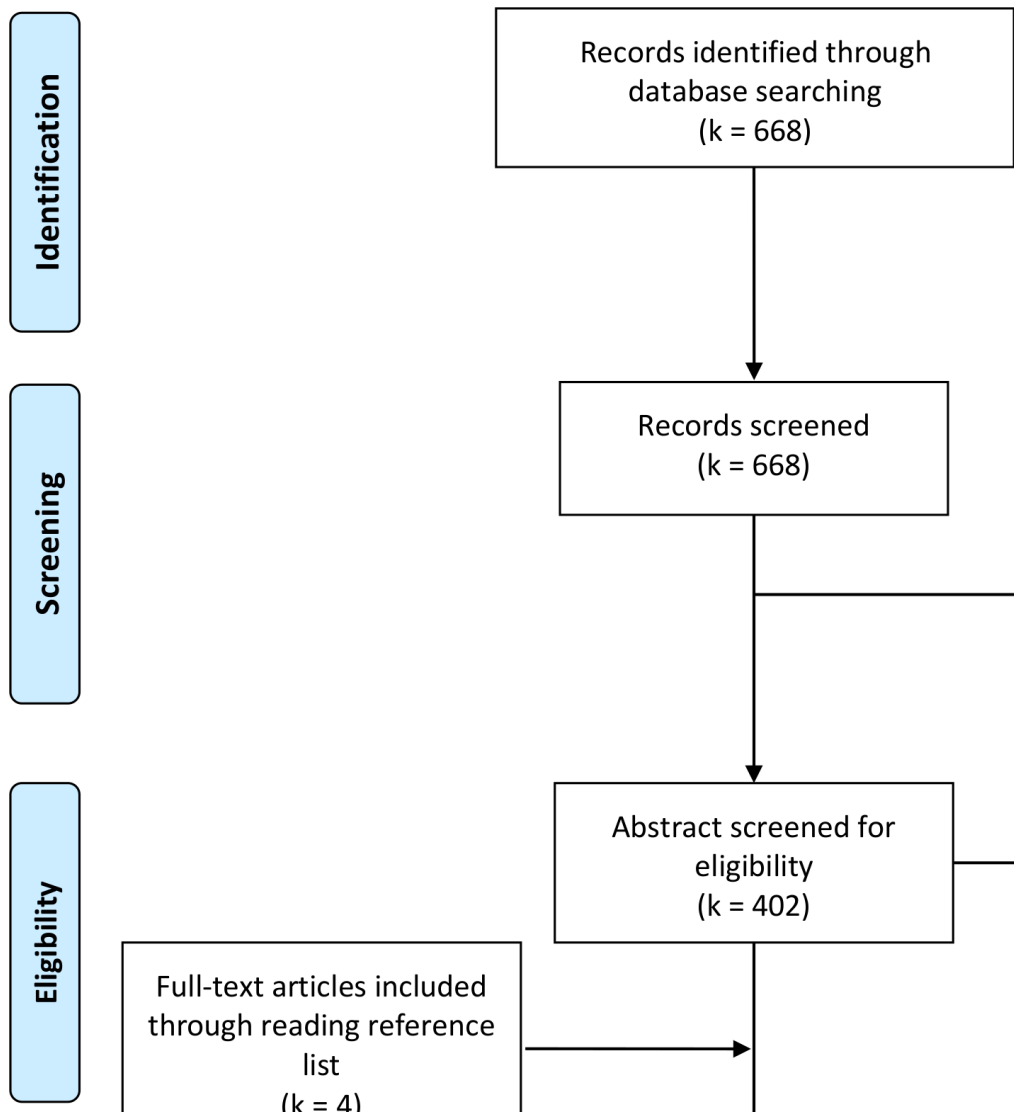

Full-text articles included through reading reference list $(k=4)$
Records excluded, after 10 year limitation $(k=266)$

Articles excluded, with reasons $(k=377)$

- Study design

- Do not match PICO-scheme

- Data missing

- Data do not match

- Not human

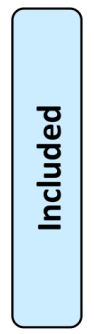

Studies included in qualitative synthesis ( $k=29)$

Studies included in quantitative synthesis (meta-analysis) $(k=29)$

Figure 1. Flow-chart describing the selection process. 
shows the flow-chart of the selection process.

\subsection{Data Extraction and Quality Assessment}

Data extraction from the studies was performed by two researchers (EH, RC). These authors extracted independently from each other the data into spreadsheets. This method was used to extract relevant data on study eligibility and content of the study (including the cooling procedure, environmental conditions, exercise protocols and outcome measures). In case of disagreement, a third researcher (RS) checked the variable in the original study and agreement was sought by consensus. The primary outcomes considered in this meta-analysis were time to finish or exhaustion, maximum speed, covered distance or PPO of a specified exercise task. Data were extracted at the end of the performance of a specified exercise. The methodological quality of the studies was assessed with the Cochrane Risk of Bias Tool [35]. Two researchers independently evaluated the 29 studies (RS, RC). A third researcher (EH) rated in case of disagreement and agreement was sought by consensus.

\subsection{Data Analysis}

Several meta-analyses and forest plot drawings were conducted using the Comprehensive Meta-Analysis software (CMA II, Biostat Inc., Englewood, NJ 07631, US). A priori it was decided to use a random-effects model because the studies under investigation were not exact replicates of each other. The DerSimonian and Laird inversed-variance method was used to calculate the weighting factors. Because individual studies reported study results in different metrics, the calculated individual study effect sizes were standardized and expressed as Hedges' $g$ to correct for overestimation of the true effect size in small study samples. The corresponding $95 \%$ confidence intervals $(95 \% \mathrm{CI}$ ) around the individual studies effect sizes as well as around the overall weighted effect were calculated. The latter indicates the range in which the mean effect size may fall, based on the universe from which this set of studies was sampled. Cohen's benchmarking for the interpretation of the effect size was followed: g lower than 0.2 (neglible effect size), g between 0.21 and 0.49 (small effect size), g between 0.50 and 0.79 (moderate effect size), and g equal to or higher than 0.8 (very high effect size) [36].

To test the Null hypothesis of heterogeneity (i.e. that all studies have a common effect size), a Cochran's Q-test was conducted and the Q-value was reported together with its corresponding degrees of freedom $(\mathrm{df}(\mathrm{Q}))$ and exact $p$-value. The significance level of this Q-test was set at 0.1 because this test is known to lack statistical power. Higgins' $I^{2}$ was calculated to express the amount of the total observed variance that can be explained by the true between studies variance rather than random sampling error and was reported as a relative number (i.e. in \%). Higgins' suggested benchmarking values for the interpretation of $\mathrm{I}^{2}$ was followed: $\mathrm{I}^{2}$ around $25 \%$ (low heterogeneity), $\mathrm{I}^{2}$ around $50 \%$ (moderate heterogeneity) and $\mathrm{I}^{2}$ around $75 \%$ or more (high heterogeneity) [37]. To 
give the reader a more absolute estimate of the distribution of possible true effect sizes on population level $\mathrm{T}^{2}$ (i.e. the variance of the distribution of true effects) and its square root (i.e. the standard deviation of the distribution of true effect sizes) were calculated. The latter allowed for the calculation of the $95 \%$ prediction intervals (95\% PI), indicating the range in which the effect-size will fall in most populations. The latter was marked as a horizontal line through the diamond representing the overall weighted mean estimate on the forest-plots.

Subgroup meta-analysis was conducted to explain a part of the observed heterogeneity only if this was plausible (i.e. in case of a sufficient number of studies). Groups were established based on the type of exercise protocols (cycling, running and functional strength) and outcomes (cycle time to exhaustion, cycle time to finish, power output, running distance, running time, running time to exhaustion, sprint time and lifted weight) used in the individual studies. Because of the low numbers of studies within the subgroups it was decided to assume a common variance. Thus, to obtain a more accurate value of $\mathrm{T}^{2}$ in the subgroup analysis, $\mathrm{T}^{2}$ was pooled and used as the common between studies variance across all the subgroups.

The likelihood for publication bias was assessed only in the overall meta-analysis using the classic fail-safe $\mathrm{N}$ and the Duval and Tweedie's trim and fill methods [38].

\section{Results}

\subsection{Risk of Bias Analysis}

Figure 2 depicts the Risk of Bias results of each included study and Figure 3 demonstrates the overview of all included studies for this analysis. Blinding of the participants was not possible in these studies. However, it was not always clear if other members of the research teams were blinded. Similarly, for nearly all other items (with the exception of reporting bias) the raters were uncertain. Thus, methodological issues in the studies under investigation may not be excluded. A low risk of reporting bias was observed in $90 \%$ of the analysed studies. For the remaining three studies (10\%), reporting bias remained unclear [39] [40] [41]. Additionally, it can be observed that the risk for selection bias (93\% for sequence generation and $97 \%$ for allocation concealment) detection bias ( $100 \%$ for blinding of outcome assessment) and other bias (100\%) remained unclear.

\subsection{Study Characteristics}

In the present work, the results of eight studies with healthy and active volunteers $(\mathrm{k}=91)$, four studies with team-sport players $(\mathrm{k}=49)$, eight studies with moderately trained $(k=57)$ and nine studies with well trained volunteers $(k=$ $81)$ were included in different meta-analyses. Hence, a total of 278 participants $(\mathrm{k}=272$ males and $\mathrm{k}=6$ females [42] [43]) were included. The mean environmental temperature in the included studies was $32.3^{\circ} \mathrm{C} \pm 2.2^{\circ} \mathrm{C}$ and mean relative humidity $53.9 \% \pm 13.5 \%$. The used exercise testing-protocols varied between 


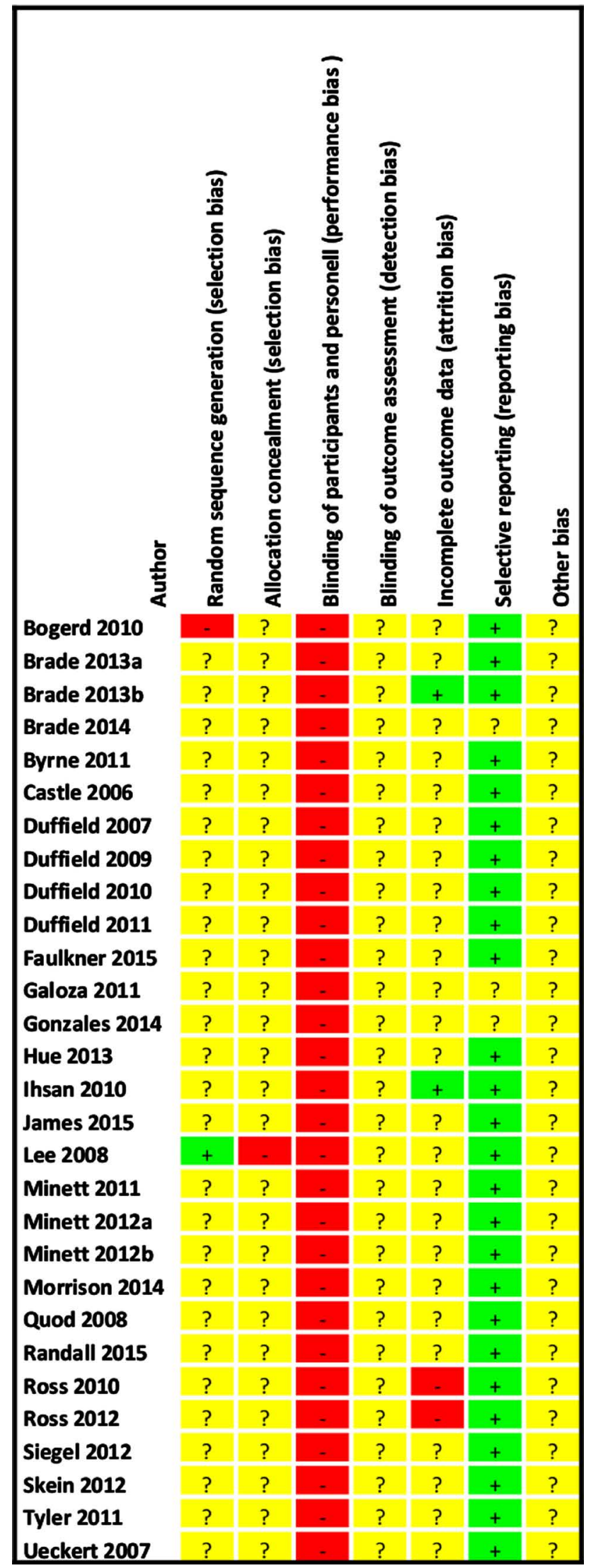

Figure 2. Risk of bias graph for each included study. 


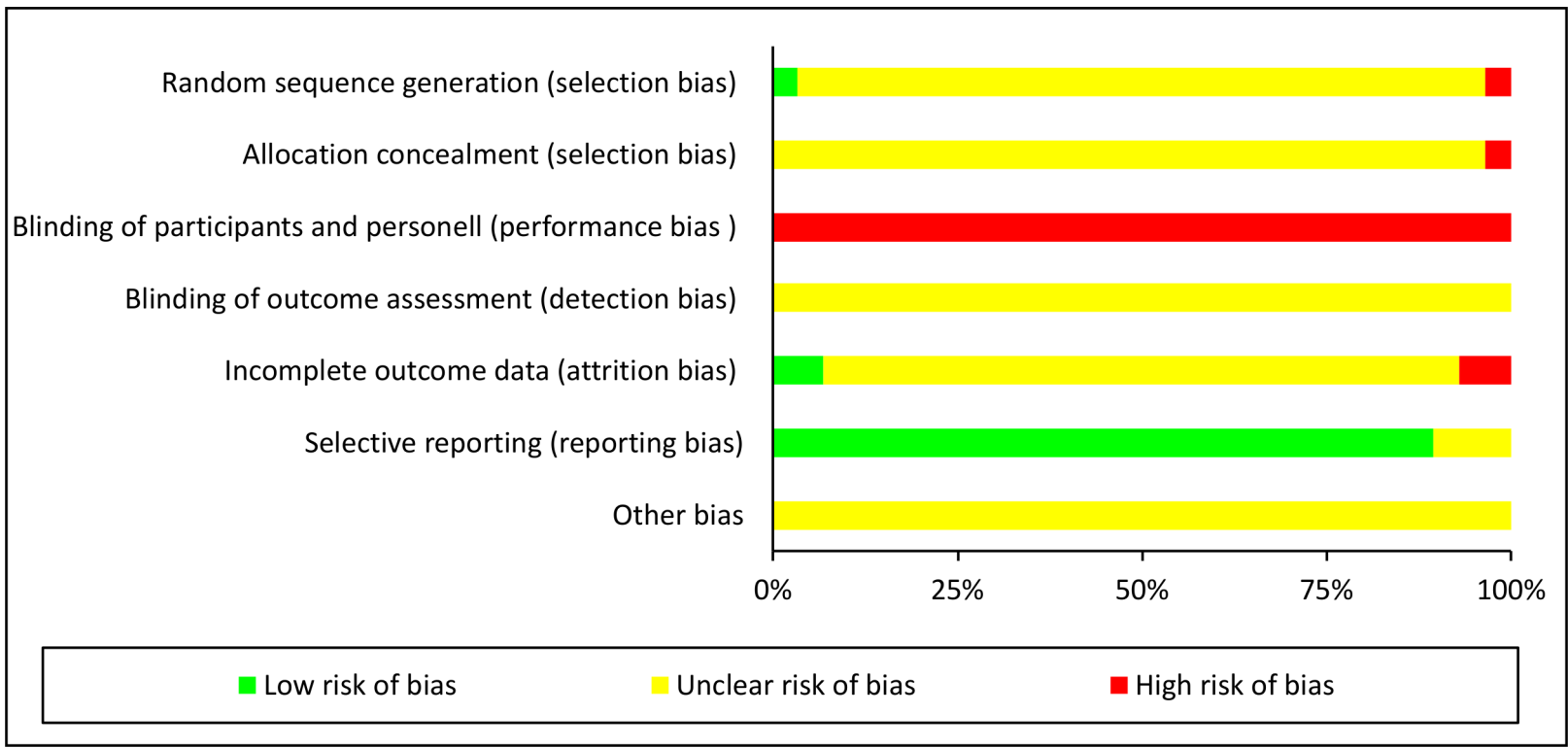

Figure 3. Risk of bias summary for all included studies.

endurance cycling $(\mathrm{k}=11)$ or running $(\mathrm{k}=5)$, repeated cycling $(\mathrm{k}=3)$ or running $(\mathrm{k}=6)$ sprints and sport specific tasks $(\mathrm{k}=4)$. Table 1 depicts a summary of the included studies.

External cooling techniques comprised of cooling vests $(\mathrm{k}=4)$, local ice applications $(\mathrm{k}=4)$, cold-water immersion ( $\mathrm{CWI} ; \mathrm{k}=3)$, combinations of external cooling $(\mathrm{k}=11)$ and whole-body cryotherapy (WBC; $\mathrm{k}=1)$. In total, 23 external cooling data sets were extracted from 21 studies.

Internal cooling techniques comprised of ingesting cold water $(\mathrm{k}=3)$ and crushed ice/ice slurry/slushies $(\mathrm{k}=4)$. In total, seven internal cooling data sets were extracted from seven studies. Mixed-method cooling comprised of the combination of ingesting ice slushies and waring cooling vests $(\mathrm{k}=3)$ or ice towel applications $(\mathrm{k}=2)$. In total, five mixed-method cooling data sets were extracted from five studies comprising of ingesting ice/drinking slushies and wearing cooling vests [39] [44] [45] or using ice towels [30] [46].

\subsection{External-Cooling vs. Non-Cooling Strategies}

In these analysis, the effects of external cooling was studied on following outcome variables: PPO $(k=5)$ [25] [39] [40] [41] [47], time to exhaustion $(k=8)$ [5] [15] [24] [48] [49] [50] [51] [52], covered distance $(k=5)$ [26] [27] [42] [53] [54] and speed $(k=3)$ [55] [56] [57]. Six studies investigated the effects of pre-cooling on endurance cycling [5] [24] [41] [47] [48] [49], five studies on endurance running [15] [50] [51] [52] [55], two on repeated cycle sprints [25] [39], five studies on repeated running sprints [26] [27] [53] [54] [57] and three studies on sport specific performance [40] [42] [56]. Authors used ice vests [24] [39] [50] [52], local cooling applications [25] [40] [50] [51], CWI [15] [26] [48] and combinations of external cooling methods [5] [27] [41] [42] [47] [49] [53] [54] [55] [56] [57]. 
Table 1. Summary of the included studies for the meta-analyses.

\begin{tabular}{|c|c|c|c|c|c|c|c|c|}
\hline \multirow{2}{*}{ Authors } & \multirow{2}{*}{ Sample size } & \multirow{2}{*}{ Cooling method } & \multirow{2}{*}{ Cooling duration } & \multicolumn{2}{|c|}{$\begin{array}{l}\text { Environmental } \\
\text { conditions }\end{array}$} & \multirow{2}{*}{ Exercise protocol } & \multirow{2}{*}{ Outcome } & \multirow{2}{*}{$\begin{array}{l}p \text {-value for } \\
\text { cooling vs. } \\
\text { control }\end{array}$} \\
\hline & & & & $\begin{array}{l}\text { Temp } \\
\left({ }^{\circ} \mathrm{C}\right)\end{array}$ & $\begin{array}{l}\mathrm{RH} \\
(\%)\end{array}$ & & & \\
\hline $\begin{array}{l}\text { Bogerd et al. } \\
(2010)\end{array}$ & $\begin{array}{l}\mathrm{k}=8 \\
\text { males }\end{array}$ & Ice vest & $45 \mathrm{~min}$ & 29 & 80 & $\begin{array}{l}\text { Endurance cycling at } \\
65 \% \mathrm{VO}_{2 \max }\end{array}$ & $\begin{array}{l}\text { Cycle time to } \\
\text { exhaustion }\end{array}$ & $<0.001$ \\
\hline $\begin{array}{l}\text { Brade et al. } \\
(2013)^{\mathrm{b}}\end{array}$ & $\mathrm{k}=10$ males & Slushy \& cooling vest & $\begin{array}{l}\text { Ingesting } 7 \mathrm{~g} / \mathrm{kg} \text { ice every } 10 \mathrm{~min} \\
\text { up to } 30 \mathrm{~min} \& 8 \mathrm{~min} \text { (vest) }\end{array}$ & 35 & 58 & $\begin{array}{l}\text { Repeated cycling } \\
\text { sprint for } 70 \mathrm{~min}\end{array}$ & $\mathrm{PPO}$ & 0.60 \\
\hline $\begin{array}{l}\text { Brade et al. } \\
\text { (2014) }\end{array}$ & $\mathrm{k}=12$ males & $\begin{array}{l}\text { Ice Vest \& Slushy. } \\
\text { + Ice Vest. } \\
+ \text { Slushy }\end{array}$ & $\begin{array}{l}\text { Vest worn and ingesting } 7 \mathrm{~g} / \mathrm{kg} \\
\text { ice for max. } 30 \mathrm{~min} .+ \text { vest worn } \\
\text { for max. } 30 \mathrm{~min} .+ \text { ingesting } 7 \\
\mathrm{~g} / \mathrm{kg} \text { ice up to } 30 \mathrm{~min} .\end{array}$ & 35 & 60 & $\begin{array}{l}\text { Repeated cycling } \\
\text { sprint for } 70 \mathrm{~min}\end{array}$ & $\mathrm{PPO}$ & $\begin{array}{l}0.22+ \\
0.28+ \\
0.28\end{array}$ \\
\hline $\begin{array}{l}\text { Byrne et al. } \\
\text { (2011) }\end{array}$ & $\begin{array}{l}\mathrm{k}=7 \\
\text { males }\end{array}$ & Ingesting cold water & $\begin{array}{l}\text { Ingesting } 900 \mathrm{ml} \text { of cold water } \\
\left(2^{\circ} \mathrm{C}\right) \text { during } 35 \mathrm{~min}\end{array}$ & 32 & 60 & $\begin{array}{l}30 \text { min self-paced } \\
\text { cycling trial }\end{array}$ & $\mathrm{PPO}$ & 0.04 \\
\hline $\begin{array}{l}\text { Castle et al. } \\
\text { (2006) }\end{array}$ & $\mathrm{k}=12$ males & Ice packs on upper legs & $20 \mathrm{~min}$ & 34 & 52 & $\begin{array}{l}\text { Intermittent cycling } \\
\text { sprint for } 20 \times 5 \mathrm{sec}\end{array}$ & $\mathrm{PPO}$ & 0.01 \\
\hline $\begin{array}{l}\text { Duffield et al. } \\
(2007)\end{array}$ & $\begin{array}{l}\mathrm{k}=9 \\
\text { males }\end{array}$ & CWI & $15 \& 10 \mathrm{~min}$ & 32 & 30 & $\begin{array}{l}\text { Intermittent sprint } \\
\text { protocol for } 2 \times 30 \\
\text { min }\end{array}$ & $\begin{array}{l}\text { Running } \\
\text { distance }\end{array}$ & 0.33 \\
\hline $\begin{array}{l}\text { Duffield et al. } \\
(2009)\end{array}$ & $\begin{array}{l}\mathrm{k}=7 \\
\text { males }\end{array}$ & $\begin{array}{l}\text { Cooling vest \& cold towels to } \\
\text { the neck \& ice packs on the } \\
\text { upper legs }\end{array}$ & $20 \mathrm{~min}$ & 32 & 44 & $\begin{array}{l}\text { Intermittent sprint } \\
\text { exercise for } 30 \mathrm{~min}\end{array}$ & $\begin{array}{l}\text { Running } \\
\text { distance }\end{array}$ & 0.001 \\
\hline $\begin{array}{l}\text { Duffield et al. } \\
(2010)\end{array}$ & $\begin{array}{l}\mathrm{k}=8 \\
\text { males }\end{array}$ & CWI & $20 \mathrm{~min}$ & 33 & 50 & $\begin{array}{l}\text { Cycle time trial for } \\
40 \mathrm{~min}\end{array}$ & $\mathrm{PPO}$ & 0.01 \\
\hline $\begin{array}{l}\text { Faulkner et al. } \\
(2015)\end{array}$ & $\mathrm{k}=10$ males & Cooling vest \& cooling sleeves & $9 \mathrm{~min}$ & 35 & 50 & $\begin{array}{l}\text { Cycling } 60 \mathrm{~min} \text { at } \\
75 \% \mathrm{~W}_{\max } \text { as fast as } \\
\text { possible }\end{array}$ & $\mathrm{PPO}$ & 0.03 \\
\hline $\begin{array}{l}\text { Galoza et al. } \\
(2011)\end{array}$ & $\mathrm{k}=16$ males & Ice bags on the upper arm & $3 \times 1 \mathrm{~min}$ & $\mathrm{n} / \mathrm{a}$ & $\mathrm{n} / \mathrm{a}$ & $\begin{array}{l}4 \text { sets of biceps curls } \\
\text { at } 70 \% \text { of } 1 \mathrm{RM}\end{array}$ & Weight lifted & 0.05 \\
\hline $\begin{array}{l}\text { Gonzales et al. } \\
\text { (2014) }\end{array}$ & $\mathrm{k}=10$ males & Cooling vest $\&$ headband & $5 \& 15 \mathrm{~min}$ & 30 & 79 & $\begin{array}{l}\text { Cycle time trial for } \\
20 \mathrm{~min}\end{array}$ & $\mathrm{PPO}$ & 0.01 \\
\hline $\begin{array}{l}\text { Hue et al. } \\
(2013)\end{array}$ & $\begin{array}{l}\mathrm{k}=5 \\
\text { males and } \mathrm{k} \\
=4 \text { females }\end{array}$ & Ingesting ice water & $\begin{array}{l}\text { Ingesting } 190 \mathrm{ml} \text { water before } \\
\text { exercise }\end{array}$ & 28 & 73 & $\begin{array}{l}10 \times 100 \mathrm{~m} \\
\text { swimming }\end{array}$ & $\begin{array}{l}\text { Swimming } \\
\text { time }\end{array}$ & 0.66 \\
\hline $\begin{array}{l}\text { Ihsan et al. } \\
\text { (2010) }\end{array}$ & $\begin{array}{l}\mathrm{k}=7 \\
\text { males }\end{array}$ & Ingesting crushed ice & $\begin{array}{l}\text { Ingesting } 6.8 \mathrm{~g} / \mathrm{kg} \text { ice within } 30 \\
\mathrm{~min}\end{array}$ & 30 & 75 & $\begin{array}{l}\text { Cycle time trial for } \\
40 \mathrm{~min}\end{array}$ & $\mathrm{PPO}$ & 0.12 \\
\hline $\begin{array}{l}\text { James et al. } \\
\text { (2015) }\end{array}$ & $\mathrm{k}=12$ males & $\begin{array}{l}\text { Ice slurry }+ \text { Cold towels to the } \\
\text { neck and head \& water } \\
\text { immersion of hands \& cooling } \\
\text { vest \& ice packs on upper legs }\end{array}$ & $\begin{array}{l}\text { Ingesting } 7.5 \mathrm{~g} / \mathrm{kg} \text { of ice within } 20 \\
\mathrm{~min}+20 \mathrm{~min}\end{array}$ & 32 & 62 & $\begin{array}{l}\text { Incremental } \\
\text { treadmill test }\end{array}$ & $\begin{array}{l}\text { Running } \\
\text { speed }\end{array}$ & $\begin{array}{l}0.73(\mathrm{HI}) \\
0.63(\mathrm{LI})+ \\
0.27(\mathrm{HI}) \\
0.14(\mathrm{LI})\end{array}$ \\
\hline $\begin{array}{l}\text { Lee et al. } \\
(2008)\end{array}$ & $\begin{array}{l}\mathrm{k}=8 \\
\text { males }\end{array}$ & Ingesting cold drink & $\begin{array}{l}3 \text { bottles of } 300 \mathrm{ml} \text { of old water } \\
\text { within } 20 \text { min each bottle in } 2 \\
\text { min. }\end{array}$ & 35 & 69 & $\begin{array}{l}\text { Cycling at } 65 \% \\
\mathrm{VO}_{2 \max } \text { until } \\
\text { exhaustion }\end{array}$ & $\begin{array}{l}\text { Cycle time to } \\
\text { exhaustion }\end{array}$ & $<0.001$ \\
\hline $\begin{array}{l}\text { Minett et al. } \\
\text { (2011) }\end{array}$ & $\mathrm{k}=10$ males & $\begin{array}{l}\text { Cold towels to the neck and } \\
\text { head \& water immersion of } \\
\text { hands \& cooling vest \& ice } \\
\text { packs on upper legs }\end{array}$ & $20 \mathrm{~min}$ & 33 & 33 & $\begin{array}{l}\text { Intermittent sprint } \\
\text { protocol for } 2 \times 35 \\
\text { min }\end{array}$ & $\begin{array}{l}\text { Running } \\
\text { distance }\end{array}$ & 0.003 \\
\hline
\end{tabular}




\section{Continued}

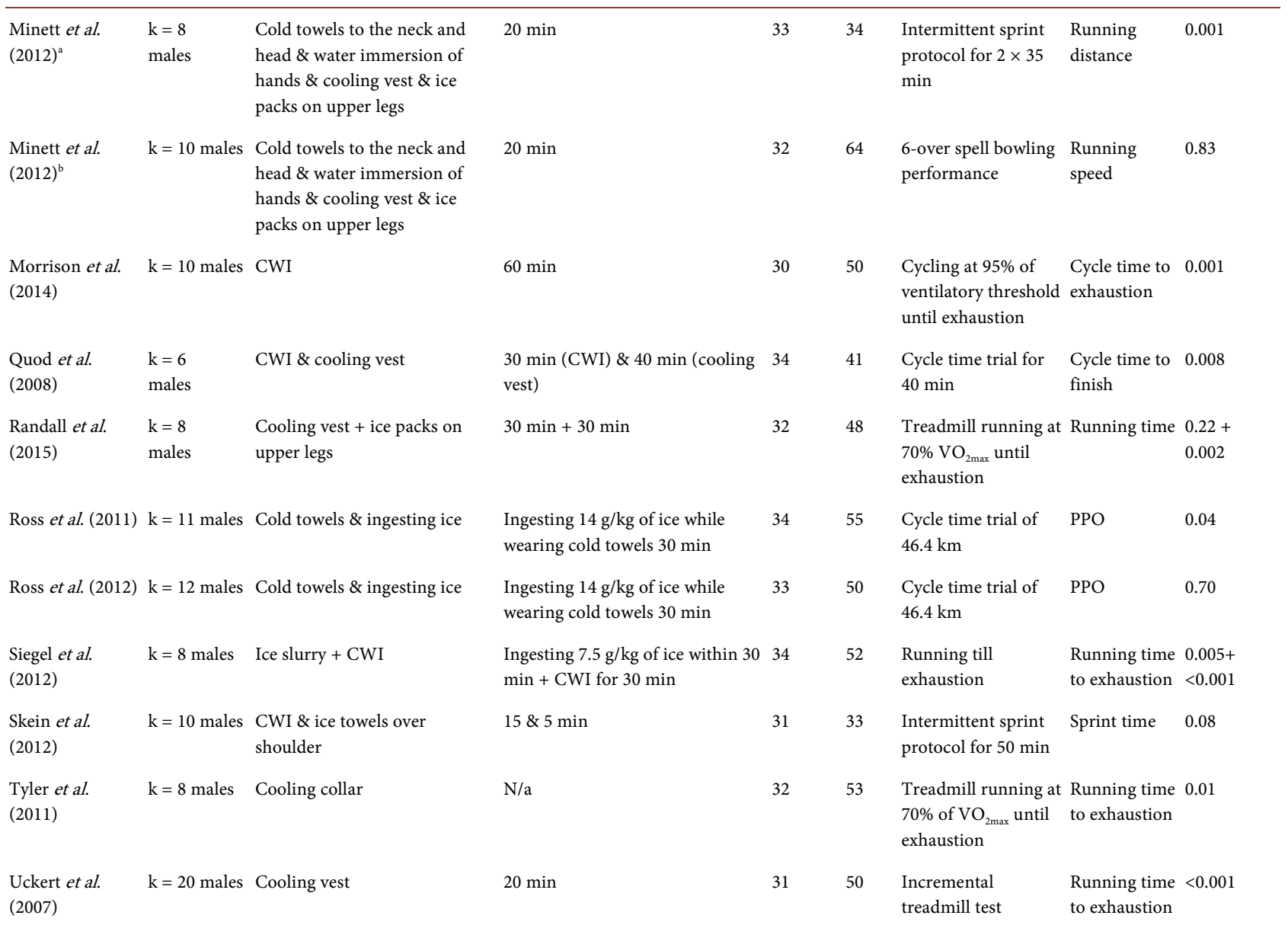

$\&=$ indicates combinations within a cooling intervention; + = indicates additional cooling intervention within one study; PPO = peak power output; CWI =

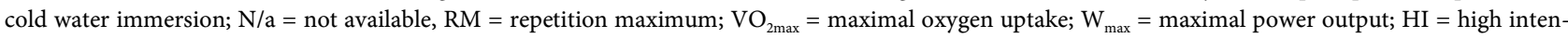
sity, $\mathrm{LI}=$ low intensity.

To test the hypothesis that external pre-cooling had an enhancing effect on performance parameters, an overall meta-analysis was conducted. This calculation showed that external pre-cooling techniques had a moderate but statistical significant effect on performance output compared to non-cooling strategies (Hedges' $g=0.49$ [95\% CI: 0.33 to 0.64]), which can be observed in Figure 4. The observed heterogeneity was high and statistically significant $(\mathrm{Q}=77.0$; $\left.\operatorname{df}(\mathrm{Q})=22 ; p<0.001 ; \mathrm{I}^{2}=71.4 \%\right)$ suggesting that about $71.4 \%$ of the variance on observed effects reflects variance in true effects. The remaining $28.6 \%$ can be attributed to random or sampling error and would probably disappear in case of very large sample sizes. The $95 \%$ prediction interval, indicating in which range the effect size in most populations will fall, ranged from -0.17 to 1.14 Hedges' g. To assess the risk for publication bias in this overall meta-analysis, a classic fail-safe $\mathrm{N}$ test was conducted and showed that 729 studies would be needed to bring $p$-value to $>0.05$. In addition, the Duval and Tweedie's trim and fill method revealed that only two small studies were missing to obtain funnel plot symmetry (Figure 5). If these two studies would be considered in the meta-analysis, the 
Study name

Bogerd et al., 2010
Brade et al., 2014
Castle et al., 2006
Duffield et al., 2007
Duffield et al., 2009
Duffield et al., 2010
Duffield et al., 2011
Faulkner et al., 2015
Galoza et al., 2011
Gonzales et al., 2014
James et al., 2015_High intensity
James et al., 2015_Low intensity
Minett et al., 2011
Minett et al., 2012a
Minett et al., 2012b
Morrison et al., 2014
Quod et al., 2008
Randall et al., 2015_packs
Randall et al., 2015_vests
Siegel et al., 2011
Skein et al., 2012
Tyler et al., 2011
Ueckert et al., 2007
Overall weighted effect

Statistics for each study

\begin{tabular}{|c|c|c|c|c|c|c|}
\hline $\begin{array}{l}\text { Hedges's } \\
\text { g }\end{array}$ & $\begin{array}{l}\text { Standard } \\
\text { error }\end{array}$ & Variance & $\begin{array}{l}\text { Lower } \\
\text { limit }\end{array}$ & $\begin{array}{l}\text { Upper } \\
\text { limit }\end{array}$ & Z-Value & p-Value \\
\hline 1.202 & 0.337 & 0.113 & 0.542 & 1.862 & 3.569 & 0.000 \\
\hline 0.226 & 0.211 & 0.045 & -0.188 & 0.640 & 1.071 & 0.284 \\
\hline 0.297 & 0.123 & 0.015 & 0.056 & 0.538 & 2.413 & 0.016 \\
\hline 0.228 & 0.237 & 0.056 & -0.236 & 0.693 & 0.963 & 0.335 \\
\hline 1.129 & 0.346 & 0.119 & 0.452 & 1.806 & 3.267 & 0.001 \\
\hline 0.696 & 0.278 & 0.077 & 0.151 & 1.242 & 2.502 & 0.012 \\
\hline 0.382 & 0.254 & 0.065 & -0.117 & 0.881 & 1.501 & 0.133 \\
\hline 0.274 & 0.132 & 0.017 & 0.015 & 0.533 & 2.070 & 0.038 \\
\hline 0.984 & 0.504 & 0.254 & -0.003 & 1.971 & 1.954 & 0.051 \\
\hline 0.322 & 0.133 & 0.018 & 0.061 & 0.584 & 2.419 & 0.016 \\
\hline 0.168 & 0.155 & 0.024 & -0.136 & 0.473 & 1.085 & 0.278 \\
\hline 0.230 & 0.157 & 0.025 & -0.077 & 0.537 & 1.467 & 0.142 \\
\hline 0.764 & 0.260 & 0.068 & 0.254 & 1.274 & 2.938 & 0.003 \\
\hline 0.481 & 0.150 & 0.023 & 0.186 & 0.776 & 3.198 & 0.001 \\
\hline-0.046 & 0.224 & 0.050 & -0.485 & 0.393 & -0.206 & 0.837 \\
\hline 0.889 & 0.272 & 0.074 & 0.356 & 1.421 & 3.270 & 0.001 \\
\hline 0.876 & 0.331 & 0.109 & 0.228 & 1.524 & 2.650 & 0.008 \\
\hline 0.566 & 0.180 & 0.032 & 0.213 & 0.919 & 3.143 & 0.002 \\
\hline 0.334 & 0.277 & 0.076 & -0.208 & 0.876 & 1.209 & 0.227 \\
\hline 1.345 & 0.356 & 0.127 & 0.646 & 2.043 & 3.772 & 0.000 \\
\hline-0.547 & 0.314 & 0.099 & -1.162 & 0.069 & -1.742 & 0.082 \\
\hline 0.352 & 0.146 & 0.021 & 0.066 & 0.638 & 2.412 & 0.016 \\
\hline 1.081 & 0.123 & 0.015 & 0.841 & 1.322 & 8.812 & 0.000 \\
\hline 0.485 & 0.080 & 0.006 & 0.328 & 0.641 & 6.067 & 0.000 \\
\hline
\end{tabular}

\section{Hedges's g and $95 \% \mathrm{Cl}$}

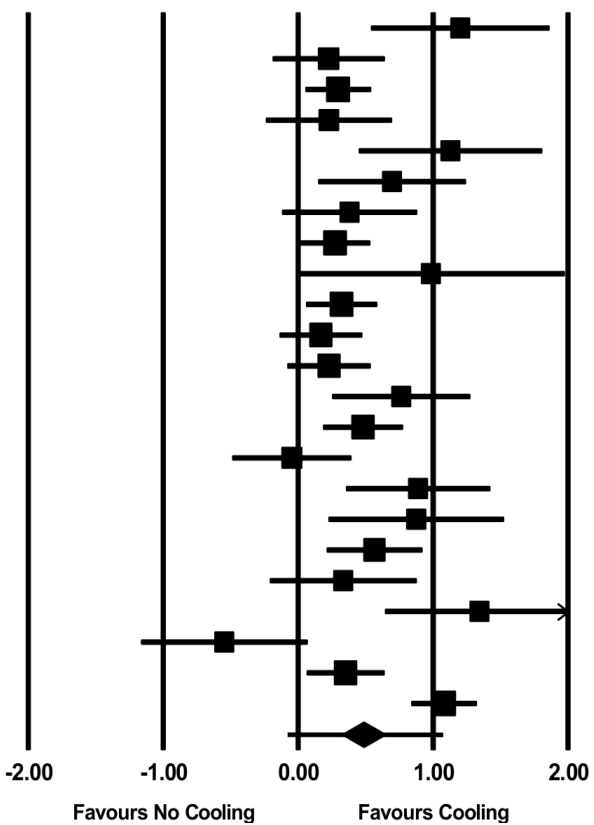

Figure 4. Forest plot of the meta-analysis illustrating the overall weighted effect of external cooling versus no cooling on performance parameters (The diamond on the bottom of the forest-plot represents the overall weighted estimate while the horizontal line through the diamond represents the $95 \%$ prediction interval).

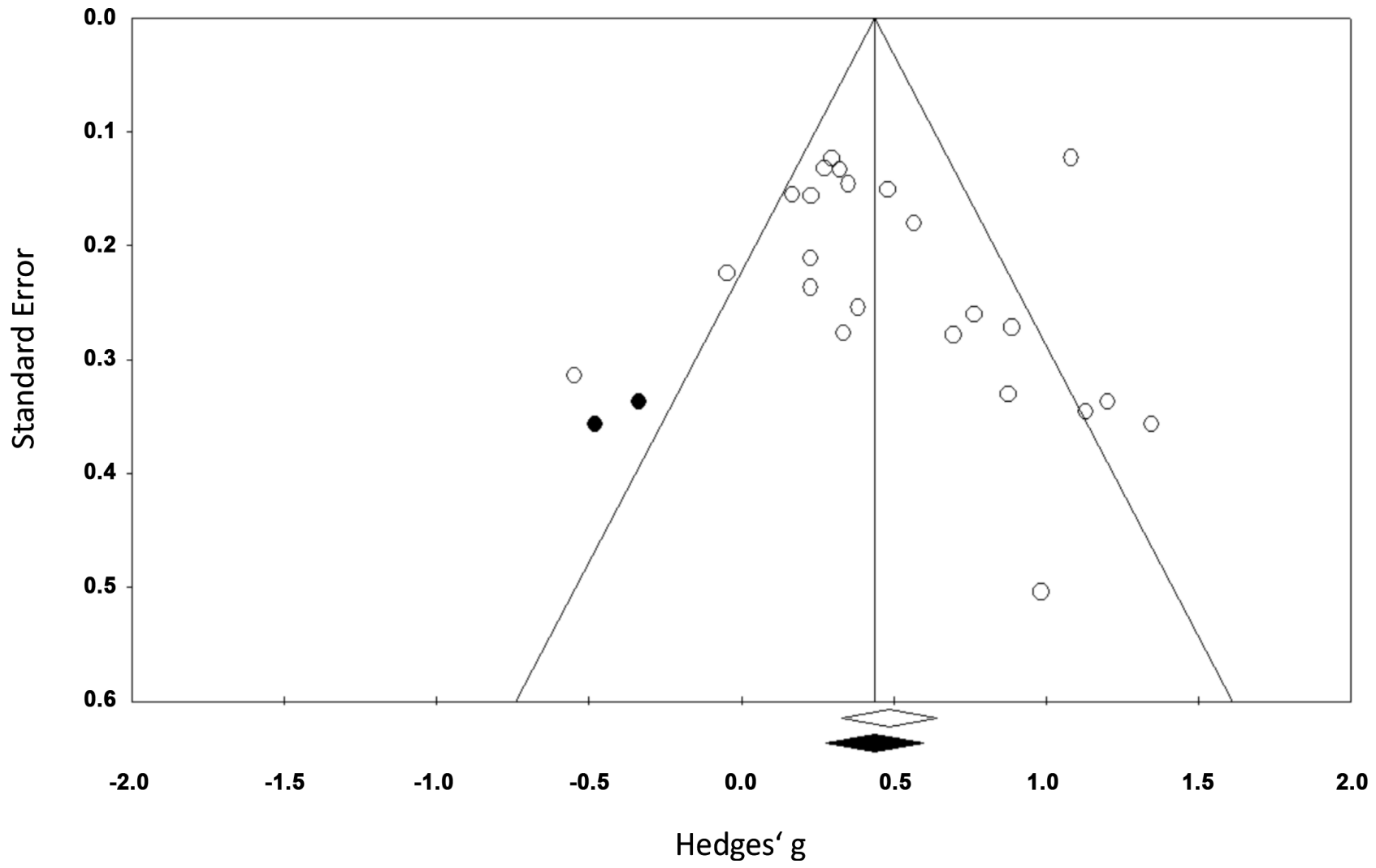

Figure 5. Funnel plot of the included studies using external-cooling strategies (The white diamond indicates the actual effect size while the black diamond indicates the adjusted effect size after adding the $\mathrm{k}=2$ missing studies). 
overall weighted effect-size would yield an adjusted value of Hedges' $\mathrm{g}=0.43$ [95\% CI: 0.28 to 0.58$]$ ]).

In an effort to explain a part of the observed high heterogeneity in this overall meta-analysis, different subgroup meta-analyses were conducted by stratifying studies based on their type of exercise protocols (cycling, running and functional strength) and outcomes (cycle time to exhaustion, cycle time to finish, power output, running distance, running time, running time to exhaustion, sprint time, running speed and lifted weight). Pre-cooling was effective for enhancing performance characteristics in humans for both cycling (Hedges' $\mathrm{g}=0.53$, [95\% CI: 0.26 to 0.80$] ; \mathrm{Q}=14.7, \mathrm{df}(\mathrm{Q})=7, p=0.037 ; \mathrm{I}^{2}=52.5 \%$ ) and running (Hedges' $\mathrm{g}$ $=0.45$, [95\% CI: 0.25 to 0.65$\left.] ; \mathrm{Q}=59.9, \mathrm{df}(\mathrm{Q})=13 ; p<0.001 ; \mathrm{I}^{2}=78.3 \%\right)$ exercises.

Pre-cooling had very strong, positive and statistical significant effects on cycle time to exhaustion (Hedges' $\mathrm{g}=1.02$; $[95 \% \mathrm{CI}=0.53$ to 1.51$]$ ), cycling time to finish (Hedges' $\mathrm{g}=0.88$; [95\% CI: 0.23 to 1.52$]$ ) and running time to exhaustion (Hedges' $\mathrm{g}=0.82$; [95\% CI: 0.53 to 1.11 ]), while low respectively moderate, positive and statistical significant effects of pre-cooling were observed on PPO (Hedges' $\mathrm{g}=0.33$; [95\% CI: 0.11 to 0.55$]$ ), running distance (Hedges' $\mathrm{g}=0.54$; [95\% CI: 0.28 to 0.80$]$ ) and running time (Hedges' $\mathrm{g}=0.48$; [95\% CI: 0.08 to $0.88])$. In these subgroups heterogeneity disappeared except for the "running time to exhaustion" subgroup where heterogeneity remained high $(\mathrm{Q}=17.0$, $\left.\mathrm{df}(\mathrm{Q})=2, p<0.001 ; \mathrm{I}^{2}=88.2\right)$. External pre-cooling had no main effect on sprint time (Hedges' $\mathrm{g}=-0.55$; [95\% CI: -1.16 to 0.07 ]), running speed (Hedges' $\mathrm{g}=0.15$; [95\% CI: -0.04 to 0.35$]$ ) and lifted weight (Hedges' $\mathrm{g}=0.98$; [95\% CI: -0.003 to 1.97$])$.

\subsection{Internal-Cooling vs. Non-Cooling Strategies}

Seven studies revealed the effect of internal pre-cooling on performance parameters [15] [28] [29] [39] [43] [55] [58] with one study investigating the effect on performance using a 70 min repeated cycling sprint protocol [39], one study using an endurance swimming task [43], two studies using endurance running [15] [55] and three studies using endurance cycling tasks [28] [29] [58]. Four studies examined the effects of ingesting ice [15] [29] [39] [55] on performance while three studies used cold water [28] [43] [58] as a cooling intervention. The overall weighted mean estimate showed that internal cooling has a moderate and positive, albeit no statistical significant effect compared to the control condition on performance parameters (Hedges' $g=0.55$; [95\% CI: -0.01 to 1.11]) (Figure 6). The $95 \%$ prediction interval indicating the range of possible true effect sizes in most populations ranged from -1.38 to 2.48 Hedges' g. The observed heterogeneity was high and statistically significant $\left(\mathrm{Q}=56.38\right.$, $\mathrm{df}(\mathrm{Q})=7 ; p<0.001 ; \mathrm{I}^{2}$ $=87.5 \%)$. The heterogeneity could not be explained with a subgroup analysis, according to cooling method, exercise task or performance outcome, because meaningful grouping was not plausible. 


\subsection{Mixed-Method Internal and External Cooling vs. Non-Cooling Strategies}

Five studies examined the effects of mixed-method internal and external cooling versus non-cooling strategies on PPO and performance times [30] [39] [44] [45] [46]. Four studies investigated the effect of pre-cooling prior to a cycle sprint [39] [45] or endurance cycle trial [30] [46] while one study investigated the effect of pre-cooling on repeated sprint running times [44]. The overall weighted mean effect size between mixed method pre-cooling and the control group was very low and statistically not significant (Hedges' $g=0.07$; [95\%CI: -0.06 to $0.22])$. Heterogeneity was low $\left(\mathrm{I}^{2}=17.41 \%\right)$ and not significant $(\mathrm{Q}=4.84 ; \mathrm{df}(\mathrm{Q})$ $=4 ; p=0.30$ ). The $95 \%$ prediction interval ranged from -0.24 to 0.39 (see Figure 7). These results indicate that mixed-method pre-cooling is not superior compared to the non-cooling control group for enhancing performance. Subgroup analyses or meta-regression was not plausible to conduct due to the low number of studies included in this analysis.

\section{Discussion}

The aim of this systematic review and meta-analysis was to evaluate the effects of

$\underline{\text { Study name }}$

Brade et al., 2014 Byrne et al., 2011 Hue et al., 2013 Ihsan et al., 2010 James et al., 2015_High intensity James et al., 2015_Low intensity Lee et al., 2008

Siegel et al., 2012 Overall weighted effect
Statistics for each study

\begin{tabular}{|c|c|c|c|c|c|c|}
\hline $\begin{array}{l}\text { Hedges's } \\
\text { g }\end{array}$ & $\begin{array}{l}\text { Standard } \\
\text { error }\end{array}$ & Variance & $\begin{array}{l}\text { Lower } \\
\text { limit }\end{array}$ & $\begin{array}{l}\text { Upper } \\
\text { limit }\end{array}$ & Z-Value & $\mathrm{p}$-Value \\
\hline-0.224 & 0.211 & 0.045 & -0.638 & 0.189 & -1.064 & 0.287 \\
\hline 0.478 & 0.239 & 0.057 & 0.009 & 0.947 & 1.999 & 0.046 \\
\hline 0.101 & 0.234 & 0.055 & -0.357 & 0.560 & 0.433 & 0.665 \\
\hline 0.413 & 0.269 & 0.072 & -0.113 & 0.939 & 1.538 & 0.124 \\
\hline 0.185 & 0.534 & 0.285 & -0.863 & 1.232 & 0.346 & 0.730 \\
\hline 0.252 & 0.535 & 0.287 & -0.798 & 1.301 & 0.470 & 0.638 \\
\hline 2.484 & 0.311 & 0.097 & 1.874 & 3.094 & 7.981 & 0.000 \\
\hline 0.668 & 0.236 & 0.056 & 0.205 & 1.131 & 2.830 & 0.005 \\
\hline 0.551 & 0.285 & 0.081 & -0.009 & 1.110 & 1.930 & 0.054 \\
\hline
\end{tabular}

Hedges's g and $95 \% \mathrm{Cl}$

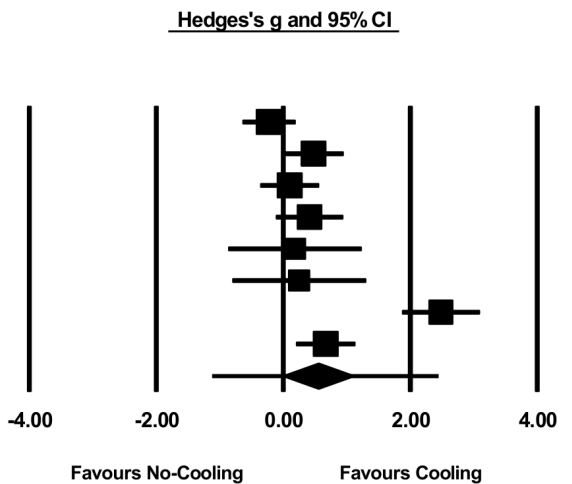

Figure 6. Forest plot of the meta-analysis illustrating the overall weighted effect of internal cooling versus no cooling on performance parameters (The diamond on the bottom of the forest-plot represents the overall weighted estimate while the horizontal line through the diamond represents the $95 \%$ prediction interval).

Study name

\begin{tabular}{lccrccrrr} 
& $\begin{array}{c}\text { Hedges's } \\
\mathbf{g}\end{array}$ & $\begin{array}{c}\text { Standard } \\
\text { error }\end{array}$ & \multicolumn{2}{c}{$\begin{array}{c}\text { Lower } \\
\text { Variance }\end{array}$} & $\begin{array}{c}\text { Upper } \\
\text { limit }\end{array}$ & $\begin{array}{l}\text { limit } \\
\text { Z-Value }\end{array}$ & p-Value \\
Brade et al., 2014 & 0.258 & 0.212 & 0.045 & -0.158 & 0.673 & 1.215 & 0.224 \\
Brade et al., 2013a & -0.105 & 0.225 & 0.050 & -0.546 & 0.335 & -0.468 & 0.640 \\
Brade et al., 2013b & -0.117 & 0.225 & 0.051 & -0.558 & 0.324 & -0.520 & 0.603 \\
Ross et al., 2011 & 0.282 & 0.139 & 0.019 & 0.009 & 0.555 & 2.022 & 0.043 \\
Ross et al., 2012 & 0.025 & 0.066 & 0.004 & -0.104 & 0.154 & 0.377 & 0.706 \\
Overall weighted effect & 0.075 & 0.069 & 0.005 & -0.061 & 0.211 & 1.078 & 0.281
\end{tabular}

Hedges's g and $95 \% \mathrm{Cl}$

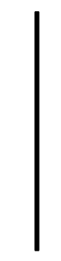

$$
-1.00
$$

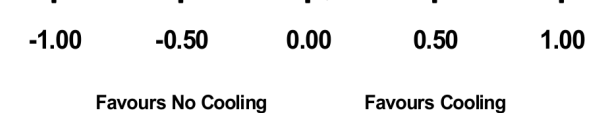

Figure 7. Forest plot of the meta-analysis illustrating the overall weighted effect of mixed-method cooling versus no cooling on performance parameters (The diamond on the bottom of the forest-plot represents the overall weighted estimate while the horizontal line through the diamond represents the $95 \%$ prediction interval). 
pre-cooling (external cooling, internal cooling and mixed-method cooling) vs. non-cooling strategies on different performance parameters in hot and humid environmental conditions. The analysis demonstrated that, prior to exercise tasks, external cooling methods had a significant better effect on performance parameters compared to non-cooling strategies. However, internal cooling and mixed method cooling strategies were not superior compared to non-cooling strategies to enhance performance.

\subsection{External-Cooling vs. Non-Cooling Strategies}

The overall weighted mean difference revealed a significant $(p<0.001)$ effect of pre-exercise external cooling applications on the performance output compared to non-cooling strategies. The observed effect size for this analysis was small (Hedges' g: 0.49). The strongest effect of pre-cooling was observed for cycling performance (Hedges' g: $0.53 ; p<0.001$ ), primarily cycle time to exhaustion ( $p<$ $0.001)$ and cycling time to finish $(p=0.008)$ under hot (ranging from 29 to $34^{\circ} \mathrm{C}$ ) and humid (ranging from 41 to $80 \%$ ) conditions. Only one study in the subgroup analysis showed a non-significant result for external pre-cooling on cycle performance [39]. The main difference to the other studies was, that Brade et al. (2014) did use a repeated sprint protocol with a 10 min break between the sets. Each set comprised of 120 maximal sprints at varying intensities, which made (self-) pacing very difficult in this protocol. These findings suggest that vasoconstriction occurred, preventing heat exchange from the body-core to the surroundings (cooling vest), resulting in non-affected core temperature changes. Similar observations were already described in previously published studies [24] [59].

Pre-cooling demonstrated to be overall significant effective for running tasks $(p<0.001)$. Open-end exercise tests, like the determination of total running distance and running time to exhaustion seem to be primarily positive affected from pre-cooling ( $p<0.001$ and $p=0.003$ ), especially under hot (ranging from $31^{\circ} \mathrm{C}$ to $35^{\circ} \mathrm{C}$ ) and humid (ranging from $30 \%$ to $53 \%$ ) conditions. However, the subgroup analysis demonstrated that it was ineffective to enhance running speed and sprint times and weight lifting. It has already been reported that decreased muscle temperature from excessive cooling can negatively influence voluntary force production and sprint performance [57]. Decreased tissue temperature has been shown to reduce nerve conduction velocity, muscle force production and muscular power [60] [61] [62]. These observations are in line with the findings of Galoza et al. (2011), demonstrating that external pre-cooling failed to significantly affect weight lifting activity in the biceps brachii muscle compared to a non-cooling strategy [40]. However, it has to be taken into account that only one study was included in the subgroup analysis of the present meta-analysis for assessing sprint time and weight lifting, respectively.

The results of this meta-analysis show some evidence that external pre-cooling methods may be effective for enhancing performance parameters with combina- 
tions of external cooling methods ( $\mathrm{k}=5$; Hedges' g range: 0.32 to 1.12$)$, CWI ( $\mathrm{k}$ $=3$; Hedges' g range: 0.69 to 1.34$)$, cooling vests $(\mathrm{k}=2$; Hedges' g range: 0.27 to $1.20)$ and local cooling applications ( $\mathrm{k}=3$; Hedges' $\mathrm{g}$ range: 0.29 to 0.56$) \mathrm{dem}$ onstrating to have the largest effect.

\subsection{Internal-Cooling vs. Non-Cooling Strategies}

The overall weighted effect indicated that internal pre-cooling is not effective vs. non-cooling strategies $(p=0.05)$ to enhance performance under hot and humid environmental conditions. As it can be observed in Figure 6, only two studies showed statistically significant effects, favouring internal cooling [15] [58]. Interestingly, these two authors investigated the effects on open-end protocols (time to exhaustion) during endurance running [15] and cycling [58]. The remaining included studies evaluated the effects on PPO [28] [29] [39] or speed factors [55] with no superior effect of pre-cooling with ice. These findings are in line with a comparable finding [32]. Other studies have already demonstrated that the effect of internal cooling is only limited. One main mechanism, which is not present after or during internal cooling methods, is the reduction of the shell temperature. This drop in temperature causes a redistribution of blood to the core, leading to a reduced heart rate [63] and a larger stroke volume [6]. However, the present study indicates that internal cooling can decrease core temperature. Reduced core temperature is well known to increase heat storage capacity [64]. However, a lower body core temperature may also reduce the participants' performance output due to central regulation mechanisms [65] because tissue temperature reductions will negatively influence the maximal loco-motor speed through reduced afferent and efferent signal transmission [66]. Muscle contractions produce heat inside the muscle, before any change in core temperature can be noticed. This muscle temperature rise increases the rate of force development of a muscle twitch with positive effects on power and speed [67] [68]. The results of this present meta-analysis are in line with comparable studies showing the efficacy of internal pre-cooling on endurance performance [32]. It has been speculated that the cooled water, while passing the oesophagus, might reduce the blood temperature in the carotid artery and this would decrease the athlete's brain temperature, leading to enhanced performance output in the heat [69] [70]. However, most research, concentrating on the effectiveness of internal cooling on performance tries to explain the mechanisms via changes in core temperature. More mechanistic (e.g. investigation of brain and muscle temperature or cutaneous vascular conductance) insights are needed to further elucidate the potential mechanisms behind this very practical pre-cooling method.

\subsection{Mixed-Method Internal and External Cooling vs. Non-Cooling Strategies}

This meta-analysis could not demonstrate a statistically significant $(p=0.28)$ 
overall weighted effect of mixed-method pre-cooling on performance output. However, it has to be mentioned, that the included studies used acclimatization periods prior to the pre-cooling method (for the experimental and control group). Acclimatization to hot environmental conditions has been demonstrated to be a key component for reducing the negative effects of heat strain [19]. Brade et al. (2013) concluded that pre-cooling alone is unnecessary if athletes are heat acclimated [44]. Therefore, an increase of the heat storage capacity seems to be more effective with acclimatisation to the heat than pre-cooling the body core and shell. Acclimatisation to the heat has various physiological advantages to enhance performance. One main factor is that the increase in core and skin temperature is attenuated through increased sweat rates [71]. Evaporative heat loss is the most effective way to release heat from the body [10]. Furthermore, the increased heart rate is also attenuated because of the increasing stroke volume during acclimatisation [71]. This might be one explanation why a positive effect of pre-cooling can rather be seen in aerobic exercises than in anaerobic exercise or power outputs. Muscle power depends strongly on the actual muscle temperature. Published data have already demonstrated that an increase in muscle temperature (through immersion in hot water) leads to higher vertical jump performances [72]. A similar positive observation has been demonstrated after warm water immersion prior to intensive cycling exercises on PPO [72]. As mixed-method cooling aims to lower tissue temperature it might be possible that cold has a negative effect on dynamic force production, as described earlier [73].

\subsection{Limitations and Future Research}

Figure 2 and Figure 3 depict that the included studies had a high or unknown risk of bias. Both the implementation and the description of the random sequence generation and the procedure of the allocation concealment would have already helped to minimize the risk of a selection bias. The blinding of participants and personnel is impossible when working with cold applications. Therefore, the high risk of performance bias is not surprising in the analysed articles. The occurrence of both detection and other bias remained unclear. Only the risk for reporting bias seems to be mainly low with only three unclear rated studies. Due to these observations, it has to be taken into account, that the results of this meta-analysis can both either underestimate or overestimate the true intervention effect of pre-cooling on performance parameters. A previously published meta-analysis in the field of cooling reported already limitations according to possibly biased results of the included studies [74]. Considering this, the authors of the current study implemented the prediction intervals in the forest plots to give the reader the possibility to observe the possible true effect sizes for each outcome in most populations. The included studies showed a broad variety of both endurance and sprint protocols, making meaningful subgroup analysis difficult or impossible (internal cooling and mixed-method cooling). Also the cooling protocols showed broad variety making direct comparisons difficult. 
Furthermore, the included studies used primarily at least well-trained, male participants for their investigation of pre-cooling. It is still unclear, if the effects of pre-cooling, obtained from the current observations, can be extrapolated to male, untrained or even female participants. Future research is strongly advised to control for the systematic error (especially by eliminating selection bias, detection bias and other bias). Although the effects of pre-cooling on performance are more relevant in field settings than in laboratory settings, researchers should also take into account that these results can be hardly replicated or even compared to each other.

\section{Conclusion}

Based on the results of this meta-analysis, external pre-exercise cooling appears to be an effective intervention to enhance performance in hot (between $29^{\circ} \mathrm{C}$ and $34^{\circ} \mathrm{C}$ ) and humid (41\% to $\left.80 \%\right)$ conditions. Endurance running and cycling tasks were primarily affected from external pre-cooling. Combinations of external cooling applications, CWI and wearing cooling vests demonstrated to have the largest effects. Internal and mixed-method cooling demonstrated to have no main effect on performance enhancement, although internal cooling might have a significant positive effect on both cycling and running endurance tasks. However, it has to be considered that the main outcome parameter for internal and mixed-method pre-cooling was PPO. The low number of studies made an evaluation of the possible effects of internal and mixed-method pre-cooling on endurance performance impossible. The high and unclear risk of bias of the included studies has to be taken into account when using the current results, although the risk for publication bias was very low.

\section{Acknowledgements}

The authors thank the "Thim van der Laan foundation" for the financial support.

\section{Conflict of Interest Statement}

The authors declare no conflict of interest.

\section{Author Contributions}

Conceived and designed the experiments: EH PC RC JT. Data extraction and quality assessment: EH RS TD JT. Risk of bias assessment: EH RS RC. Analysis of the data: EH JT. Wrote the paper: EH PC JT RC. Read and approved final version of manuscript: EH RS PC RC TD JT.

\section{References}

[1] Nybo, L., Rasmussen, P. and Sawka, M.N. (2014) Performance in the Heat-Physiological Factors of Importance for Hyperthermia-Induced Fatigue. Comprehensive Physiology, 4, 657-689. https://doi.org/10.1002/cphy.c130012 
[2] Jones, P.R., Barton, C., Morrissey, D., Maffulli, N. and Hemmings, S. (2012) Pre-Cooling for Endurance Exercise Performance in the Heat: A Systematic Review. BMC Medicine, 10, 166. https://doi.org/10.1186/1741-7015-10-166

[3] Tyler, C. and Sunderland, C. (2008) The Effect of Ambient Temperature on the Reliability of a Preloaded Treadmill Time-Trial. International Journal of Sports Medicine, 29, 812-816. https://doi.org/10.1055/s-2007-989321

[4] Van Cutsem, J., De Pauw, K., Buyse, L., Marcora, S., Meeusen, R. and Roelands, B. (2017) Effects of Mental Fatigue on Endurance Performance in the Heat. Medicine and Science in Sports and Exercise, 49, 1677-1687. https://doi.org/10.1249/MSS.0000000000001263

[5] Faulkner, S.H., Hupperets, M., Hodder, S.G. and Havenith, G. (2015) Conductive and Evaporative Precooling Lowers Mean Skin Temperature and Improves Time Trial Performance in the Heat. Scandinavian Journal of Medicine \& Science in Sports, 25, 183-189. https://doi.org/10.1111/sms.12373

[6] Gonzalez-Alonso, J., Teller, C., Andersen, S.L., Jensen, F.B., Hyldig, T. and Nielsen, B. (1999) Influence of Body Temperature on the Development of Fatigue during Prolonged Exercise in the Heat. Journal of Applied Physiology, 86, 1032-1039. https://doi.org/10.1152/jappl.1999.86.3.1032

[7] Caputa, M., Feistkorn, G. and Jessen, C. (1986) Effects of Brain and Trunk Temperatures on Exercise Performance in Goats. Pflügers Archiv, 406, 184-189. https://doi.org/10.1007/BF00586681

[8] Kunstetter, A.C., Wanner, S.P., Madeira, L.G., Wilke, C.F., Rodrigues, L.O. and Lima, N.R. (2014) Association between the Increase in Brain Temperature and Physical Performance at Different Exercise Intensities and Protocols in a Temperate Environment. Brazilian Journal of Medical and Biological Research, 47, 679-688.

[9] Nybo, L. (2012) Brain Temperature and Exercise Performance. Experimental Physiology, 97, 333-339. https://doi.org/10.1113/expphysiol.2011.062273

[10] Petrofsky, J.S., Lee, S., Patterson, C., Cole, M. and Stewart, B. (2005) Sweat Production during Global Heating and during Isometric Exercise in People with Diabetes. Medical Science Monitor, 11, CR515-CR521.

[11] Brotherhood, J.R. (2008) Heat Stress and Strain in Exercise and Sport. Journal of Science and Medicine in Sport/Sports Medicine Australia, 11, 6-19. https://doi.org/10.1016/j.jsams.2007.08.017

[12] Wingo, J.E., Low, D.A., Keller, D.M., Brothers, R.M., Shibasaki, M. and Crandall, C.G. (2010) Skin Blood Flow and Local Temperature Independently Modify Sweat Rate during Passive Heat Stress in Humans. Journal of Applied Physiology, 109, 1301-1306. https://doi.org/10.1152/japplphysiol.00646.2010

[13] James, C.A., Richardson, A.J., Watt, P.W., Willmott, A.G., Gibson, O.R. and Maxwell, N.S. (2017) Short-Term Heat Acclimation Improves the Determinants of Endurance Performance and 5-km Running Performance in the Heat. Applied Physiology, Nutrition, and Metabolism, 42, 285-294.

https://doi.org/10.1139/apnm-2016-0349

[14] Arngrimsson, S.A., Petitt, D.S., Stueck, M.G., Jorgensen, D.K. and Cureton, K.J. (2004) Cooling Vest Worn during Active Warm-Up Improves 5-km Run Performance in the Heat. Journal of Applied Physiology, 96, 1867-1874. https://doi.org/10.1152/japplphysiol.00979.2003

[15] Siegel, R., Mate, J., Watson, G., Nosaka, K. and Laursen, P.B. (2012) Pre-Cooling with Ice Slurry Ingestion Leads to Similar Run Times to Exhaustion in the Heat as Cold Water Immersion. Journal of Sports Sciences, 30, 155-165. 
https://doi.org/10.1080/02640414.2011.625968

[16] Wendt, D., van Loon, L.J. and Lichtenbelt, W.D. (2007) Thermoregulation during Exercise in the Heat: Strategies for Maintaining Health and Performance. Sports Medicine, 37, 669-682. https://doi.org/10.2165/00007256-200737080-00002

[17] Kenney, W.L. and Johnson, J.M. (1992) Control of Skin Blood Flow during Exercise. Medicine and Science in Sports and Exercise, 24, 303-312. https://doi.org/10.1249/00005768-199203000-00005

[18] Nybo, L. (2008) Hyperthermia and Fatigue. Journal of Applied Physiology, 104, 871-878. https://doi.org/10.1152/japplphysiol.00910.2007

[19] Marino, F.E. (2002) Methods, Advantages, and Limitations of Body Cooling for Exercise Performance. British Journal of Sports Medicine, 36, 89-94.

[20] Gonzalez-Alonso, J. and Calbet, J.A. (2003) Reductions in Systemic and Skeletal Muscle Blood Flow and Oxygen Delivery Limit Maximal Aerobic Capacity in $\mathrm{Hu}-$ mans. Circulation, 107, 824-830. https://doi.org/10.1161/01.CIR.0000049746.29175.3F

[21] Brooks, G.A., Hittelman, K.J., Faulkner, J.A. and Beyer, R.E. (1971) Temperature, Skeletal Muscle Mitochondrial Functions, and Oxygen Debt. The American Journal of Physiology, 220, 1053-1059. https://doi.org/10.1152/ajplegacy.1971.220.4.1053

[22] Fitts, R.H. (1994) Cellular Mechanisms of Muscle Fatigue. Physiological Reviews, 74, 49-94. https://doi.org/10.1152/physrev.1994.74.1.49

[23] Lamb, G.D. and Stephenson, D.G. (2006) Point: Lactic Acid Accumulation Is an Advantage during Muscle Activity. Journal of Applied Physiology, 100, 1410-1412. https://doi.org/10.1152/japplphysiol.00023.2006

[24] Bogerd, N., Perret, C., Bogerd, C.P., Rossi, R.M. and Daanen, H.A. (2010) The Effect of Pre-Cooling Intensity on Cooling Efficiency and Exercise Performance. Journal of Sports Sciences, 28, 771-779. https://doi.org/10.1080/02640411003716942

[25] Castle, P.C., Macdonald, A.L., Philp, A., Webborn, A., Watt, P.W. and Maxwell, N.S. (2006) Precooling Leg Muscle Improves Intermittent Sprint Exercise Performance in Hot, Humid Conditions. Journal of Applied Physiology, 100, 1377-1384. https://doi.org/10.1152/japplphysiol.00822.2005

[26] Duffield, R. and Marino, F.E. (2007) Effects of Pre-Cooling Procedures on Intermittent-Sprint Exercise Performance in Warm Conditions. European Journal of Applied Physiology, 100, 727-735. https://doi.org/10.1007/s00421-007-0468-x

[27] Duffield, R., Steinbacher, G. and Fairchild, T.J. (2009) The Use of Mixed-Method, Part-Body Pre-Cooling Procedures for Team-Sport Athletes Training in the Heat. Journal of Strength and Conditioning Research/National Strength \& Conditioning Association, 23, 2524-2532. https://doi.org/10.1519/JSC.0b013e3181bf7a4f

[28] Byrne, C., Owen, C., Cosnefroy, A. and Lee, J.K. (2011) Self-Paced Exercise Performance in the Heat after Pre-Exercise Cold-Fluid Ingestion. Journal of Athletic Training, 46, 592-599. https://doi.org/10.4085/1062-6050-46.6.592

[29] Ihsan, M., Landers, G., Brearley, M. and Peeling, P. (2010) Beneficial Effects of Ice Ingestion as a Precooling Strategy on 40-km Cycling Time-Trial Performance. International Journal of Sports Physiology and Performance, 5, 140-151. https://doi.org/10.1123/ijspp.5.2.140

[30] Ross, M.L., Garvican, L.A., Jeacocke, N.A., Laursen, P.B., Abbiss, C.R., Martin, D.T., et al. (2011) Novel Precooling Strategy Enhances Time Trial Cycling in the Heat. Medicine and Science in Sports and Exercise, 43, 123-133. https://doi.org/10.1249/MSS.0b013e3181e93210 
[31] Ross, M., Abbiss, C., Laursen, P., Martin, D. and Burke, L. (2013) Precooling Methods and Their Effects on Athletic Performance: A Systematic Review and Practical Applications. Sports Medicine, 43, 207-225. https://doi.org/10.1007/s40279-012-0014-9

[32] Wegmann, M., Faude, O., Poppendieck, W., Hecksteden, A., Frohlich, M. and Meyer, T. (2012) Pre-Cooling and Sports Performance: A Meta-Analytical Review. Sports Medicine, 42, 545-564. https://doi.org/10.2165/11630550-000000000-00000

[33] Moher, D., Liberati, A., Tetzlaff, J., Altman, D.G. and Group, P. (2009) Preferred Reporting Items for Systematic Reviews and Meta-Analyses: The PRISMA Statement. Journal of Clinical Epidemiology, 62, 1006-1012. https://doi.org/10.1016/j.jclinepi.2009.06.005

[34] Liberati, A., Altman, D.G., Tetzlaff, J., Mulrow, C., Gotzsche, P.C., Ioannidis, J.P., et al. (2009) The PRISMA Statement for Reporting Systematic Reviews and Meta-Analyses of Studies That Evaluate Healthcare Interventions: Explanation and Elaboration. BMJ, 339, b2700. https://doi.org/10.1136/bmj.b2700

[35] Higgins, J.P.T. and Green, S. (2011) Cochrane Handbook for Systematic Reviews of Interventions. Version 5.1.0. The Cochrane Collaboration.

http://handbook-5-1.cochrane.org

[36] Cohen, J. (1992) A Power Primer. Psychological Bulletin, 112, 155-159. https://doi.org/10.1037/0033-2909.112.1.155

[37] Higgins, J.P. and Thompson, S.G. (2002) Quantifying Heterogeneity in a Meta-Analysis. Statistics in Medicine, 21, 1539-1558. https://doi.org/10.1002/sim.1186

[38] Borenstein, M., Hedges, L.V., Higgins, J.P.T. and Rothstein, H.R. (2009) Introduction to Meta-Analysis (Statistics and Practice). John Wiley \& Sons, Hoboken, NJ. https://doi.org/10.1002/9780470743386

[39] Brade, C., Dawson, B. and Wallman, K. (2014) Effects of Different Precooling Techniques on Repeat Sprint Ability in Team Sport Athletes. European Journal of Sport Science, 14, S84-S91. https://doi.org/10.1080/17461391.2011.651491

[40] Galoza, P., Sampaio-Jorge, F., Machado, M., Fonseca, R. and Silva, P.A. (2011) Resistance Exercise Inter-Set Cooling Strategy: Effect on Performance and Muscle Damage. International Journal of Sports Physiology and Performance, 6, 580-584. https://doi.org/10.1123/ijspp.6.4.580

[41] Gonzales, B.R., Hagin, V., Guillot, R., Placet, V., Monnier-Benoit, P. and Groslambert, A. (2014) Self-Paced Cycling Performance and Recovery under a Hot and Highly Humid Environment after Cooling. The Journal of Sports Medicine and Physical Fitness, 54, 43-52.

[42] Duffield, R., Bird, S.P. and Ballard, R.J. (2011) Field-Based Pre-Cooling for On-Court Tennis Conditioning Training in the Heat. Journal of Sports Science \& Medicine, 10, 376-384.

[43] Hue, O., Monjo, R., Lazzaro, M., Baillot, M., Hellard, P., Marlin, L., et al. (2013) The Effect of Time of Day on Cold Water Ingestion by High-Level Swimmers in a Tropical Climate. International Journal of Sports Physiology and Performance, $\mathbf{8}$, 442-451. https://doi.org/10.1123/ijspp.8.4.442

[44] Brade, C.J., Dawson, B.T. and Wallman, K.E. (2013) Effect of Pre-Cooling on Repeat-Sprint Performance in Seasonally Acclimatised Males during an Outdoor Simulated Team-Sport Protocol in Warm Conditions. Journal of Sports Science \& Medicine, 12, 565-570.

[45] Brade, C., Dawson, B. and Wallman, K. (2013) Effect of Precooling and Acclimation 
on Repeat-Sprint Performance in Heat. Journal of Sports Sciences, 31, 779-786. https://doi.org/10.1080/02640414.2012.750006

[46] Ross, M.L., Jeacocke, N.A., Laursen, P.B., Martin, D.T., Abbiss, C.R. and Burke, L.M. (2012) Effects of Lowering Body Temperature via Hyperhydration, with and without Glycerol Ingestion and Practical Precooling on Cycling Time Trial Performance in Hot and Humid Conditions. Journal of the International Society of Sports Nutrition, 9, 55. https://doi.org/10.1186/1550-2783-9-55

[47] Duffield, R., Green, R., Castle, P. and Maxwell, N. (2010) Precooling Can Prevent the Reduction of Self-Paced Exercise Intensity in the Heat. Medicine and Science in Sports and Exercise, 42, 577-584. https://doi.org/10.1249/MSS.0b013e3181b675da

[48] Morrison, S.A., Cheung, S. and Cotter, J.D. (2014) Importance of Airflow for Physiologic and Ergogenic Effects of Precooling. Journal of Athletic Training, 49, 632-639. https://doi.org/10.4085/1062-6050-49.3.27

[49] Quod, M.J., Martin, D.T., Laursen, P.B., Gardner, A.S., Halson, S.L., Marino, F.E., et al. (2008) Practical Precooling: Effect on Cycling Time Trial Performance in Warm Conditions. Journal of Sports Sciences, 26, 1477-1487. https://doi.org/10.1080/02640410802298268

[50] Randall, C.A., Ross, E.Z. and Maxwell, N.S. (2015) Effect of Practical Precooling on Neuromuscular Function and 5-km Time-Trial Performance in Hot, Humid Conditions among Well-Trained Male Runners. Journal of Strength and Conditioning Research/National Strength \& Conditioning Association, 29, 1925-1936. https://doi.org/10.1519/JSC.0000000000000840

[51] Tyler, C.J. and Sunderland, C. (2011) Cooling the Neck Region during Exercise in the Heat. Journal of Athletic Training, 46, 61-68. https://doi.org/10.4085/1062-6050-46.1.61

[52] Uckert, S. and Joch, W. (2007) Effects of Warm-Up and Precooling on Endurance Performance in the Heat. British Journal of Sports Medicine, 41, 380-384. https://doi.org/10.1136/bjsm.2006.032292

[53] Minett, G.M., Duffield, R., Marino, F.E. and Portus, M. (2011) Volume-Dependent Response of Precooling for Intermittent-Sprint Exercise in the Heat. Medicine and Science in Sports and Exercise, 43, 1760-1769. https://doi.org/10.1249/MSS.0b013e318211be3e

[54] Minett, G.M., Duffield, R., Marino, F.E. and Portus, M. (2012) Duration-Dependant Response of Mixed-Method Pre-Cooling for Intermittent-Sprint Exercise in the Heat. European Journal of Applied Physiology, 112, 3655-3666. https://doi.org/10.1007/s00421-012-2348-2

[55] James, C.A., Richardson, A.J., Watt, P.W., Gibson, O.R. and Maxwell, N.S. (2015) Physiological Responses to Incremental Exercise in the Heat Following Internal and External Precooling. Scandinavian Journal of Medicine \& Science in Sports, 25, 190-199. https://doi.org/10.1111/sms.12376

[56] Minett, G.M., Duffield, R., Kellett, A. and Portus, M. (2012) Mixed-Method Pre-Cooling Reduces Physiological Demand without Improving Performance of Medium-Fast Bowling in the Heat. Journal of Sports Sciences, 30, 907-915. https://doi.org/10.1080/02640414.2012.679677

[57] Skein, M., Duffield, R., Cannon, J. and Marino, F.E. (2012) Self-Paced Intermittent-Sprint Performance and Pacing Strategies Following Respective Pre-Cooling and Heating. European Journal of Applied Physiology, 112, 253-266. https://doi.org/10.1007/s00421-011-1972-6

[58] Lee, J.K., Shirreffs, S.M. and Maughan, R.J. (2008) Cold Drink Ingestion Improves 
Exercise Endurance Capacity in the Heat. Medicine and Science in Sports and Exercise, 40, 1637-1644. https://doi.org/10.1249/MSS.0b013e318178465d

[59] Belani, K., Sessler, D.I., Sessler, A.M., Schroeder, M., McGuire, J., Merrifield, B., et al. (1993) Leg Heat Content Continues to Decrease during the Core Temperature Plateau in Humans Anesthetized with Isoflurane. Anesthesiology, 78, 856-863. https://doi.org/10.1097/00000542-199305000-00008

[60] Rubley, M.D., Denegar, C.R., Buckley, W.E. and Newell, K.M. (2003) Cryotherapy, Sensation, and Isometric-Force Variability. Journal of Athletic Training, 38, 113-119.

[61] Lee, J.M., Warren, M.P. and Mason, S.M. (1978) Effects of Ice on Nerve Conduction Velocity. Physiotherapy, 64, 2-6.

[62] Herrera, E., Sandoval, M.C., Camargo, D.M. and Salvini, T.F. (2010) Motor and Sensory Nerve Conduction Are Affected Differently by Ice Pack, Ice Massage, and Cold Water Immersion. Physical Therapy, 90, 581-591.

https://doi.org/10.2522/ptj.20090131

[63] Mitchell, J.B., McFarlin, B.K. and Dugas, J.P. (2003) The Effect of Pre-Exercise Cooling on High Intensity Running Performance in the Heat. International Journal of Sports Medicine, 24, 118-124. https://doi.org/10.1055/s-2003-38203

[64] Booth, J., Marino, F. and Ward, J.J. (1997) Improved Running Performance in Hot Humid Conditions Following Whole Body Precooling. Medicine and Science in Sports and Exercise, 29, 943-949.

https://doi.org/10.1097/00005768-199707000-00014

[65] Noakes, T.D. (2007) The Central Governor Model of Exercise Regulation Applied to the Marathon. Sports Medicine, 37, 374-377. https://doi.org/10.2165/00007256-200737040-00026

[66] Herrera, E., Sandoval, M.C., Camargo, D.M. and Salvini, T.F. (2011) Effect of Walking and Resting after Three Cryotherapy Modalities on the Recovery of Sensory and Motor Nerve Conduction Velocity in Healthy Subjects. Revista Brasileira de Fisioterapia, 15, 233-240. https://doi.org/10.1590/S1413-35552011000300010

[67] Ranatunga, K.W. (1982) Temperature-Dependence of Shortening Velocity and Rate of Isometric Tension Development in Rat Skeletal Muscle. The Journal of Physiology, 329, 465-483.

[68] Close, R. and Hoh, J.F. (1968) Influence of Temperature on Isometric Contractions of Rat Skeletal Muscles. Nature, 217, 1179-1180. https://doi.org/10.1038/2171179a0

[69] Siegel, R. and Laursen, P.B. (2012) Keeping Your Cool: Possible Mechanisms for Enhanced Exercise Performance in the Heat with Internal Cooling Methods. Sports Medicine, 42, 89-98. https://doi.org/10.2165/11596870-000000000-00000

[70] Mariak, Z., White, M.D., Lewko, J., Lyson, T. and Piekarski, P. (1999) Direct Cooling of the Human Brain by Heat Loss from the Upper Respiratory Tract. Journal of Applied Physiology, 87, 1609-1613. https://doi.org/10.1152/jappl.1999.87.5.1609

[71] Lind, A.R. and Bass, D.E. (1963) Optimal Exposure Time for Development of Acclimatization to Heat. Federation Proceedings, 22, 704-708.

[72] Bergh, U. and Ekblom, B. (1979) Influence of Muscle Temperature on Maximal Muscle Strength and Power Output in Human Skeletal Muscles. Acta Physiologica Scandinavica, 107, 33-37. https://doi.org/10.1111/j.1748-1716.1979.tb06439.x

[73] Crowley, G.C., Garg, A., Lohn, M.S., Van, Someren, N. and Wade, A.J. (1991) Effects of Cooling the Legs on Performance in a Standard Wingate Anaerobic Power Test. British Journal of Sports Medicine, 25, 200-203. 
[74] Hohenauer, E., Taeymans, J., Baeyens, J.P., Clarys, P. and Clijsen, R. (2015) The Effect of Post-Exercise Cryotherapy on Recovery Characteristics: A Systematic Review and Meta-Analysis. PLOS ONE, 10, e0139028.

https://doi.org/10.1371/journal.pone.0139028 


\section{Supporting Information}

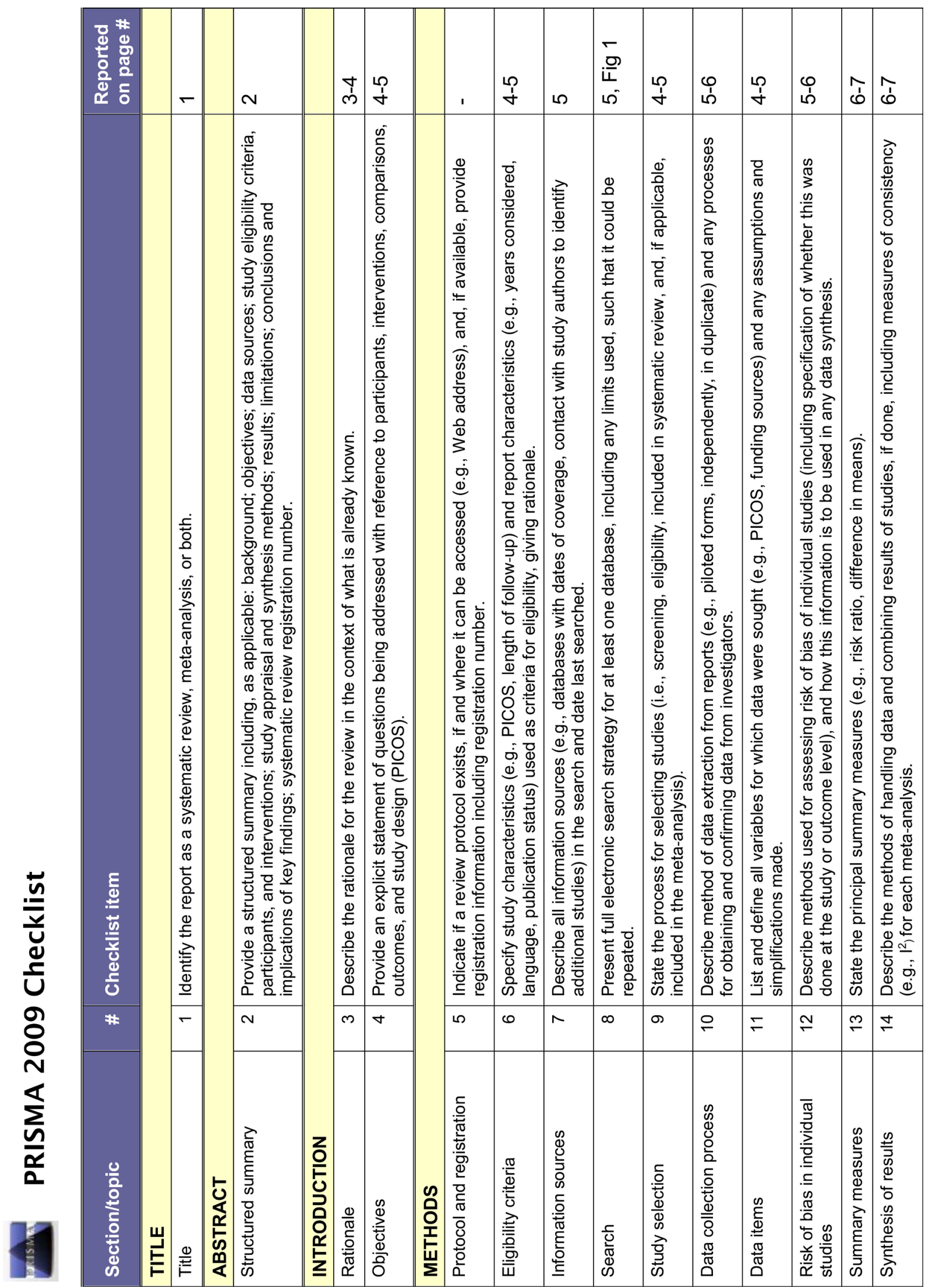




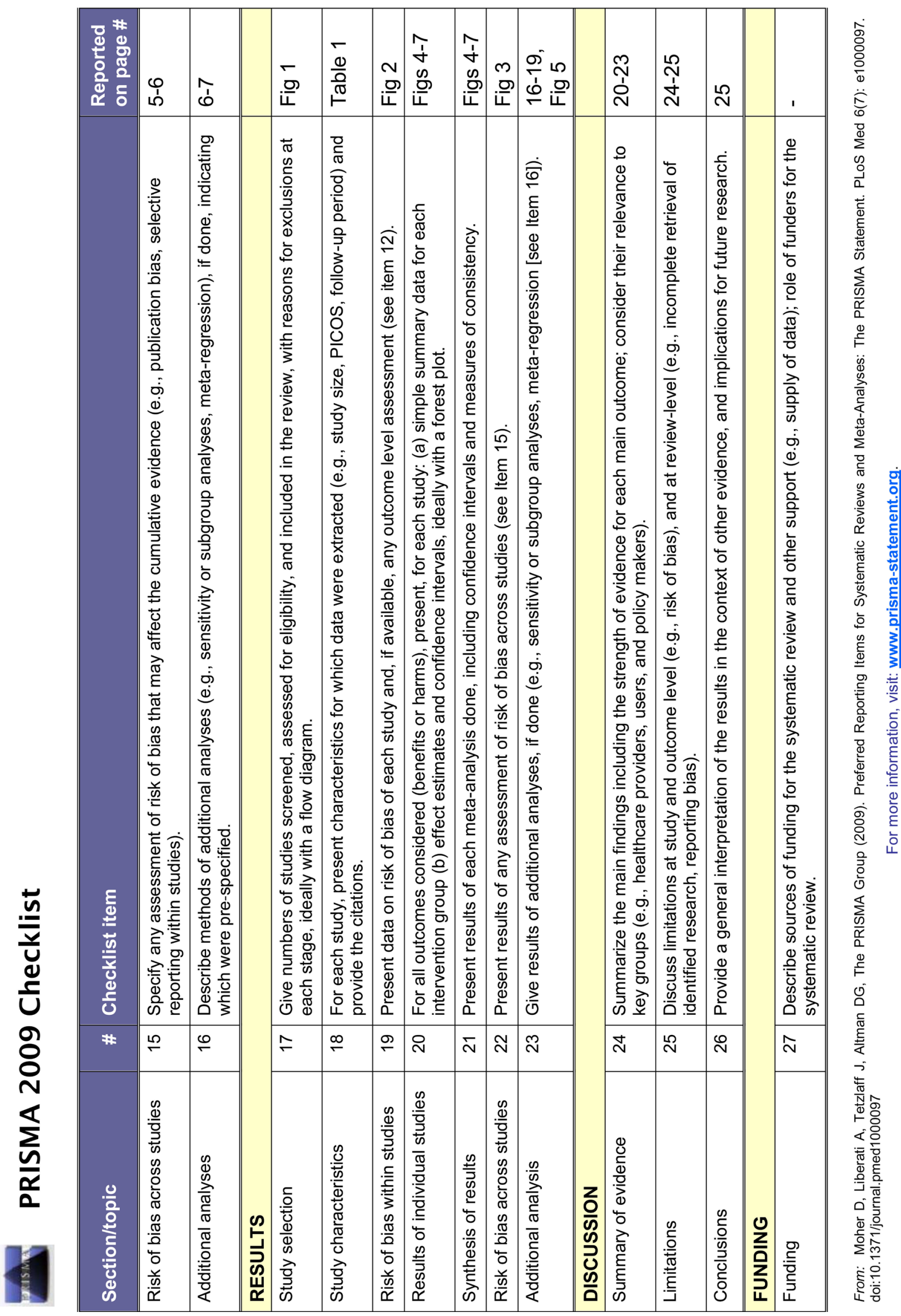

